\title{
Electromagnetic wormholes via handlebody constructions
}

\author{
Allan Greenleaf, Yaroslav Kurylev, \\ Matti Lassas and Gunther Uhlmann *
}

\begin{abstract}
Cloaking devices are prescriptions of electrostatic, optical or electromagnetic parameter fields (conductivity $\sigma(x)$, index of refraction $n(x)$, or electric permittivity $\epsilon(x)$ and magnetic permeability $\mu(x))$ which are piecewise smooth on $\mathbb{R}^{3}$ and singular on a hypersurface $\Sigma$, and such that objects in the region enclosed by $\Sigma$ are not detectable to external observation by waves. Here, we give related constructions of invisible tunnels, which allow electromagnetic waves to pass between possibly distant points, but with only the ends of the tunnels visible to electromagnetic imaging. Effectively, these change the topology of space with respect to solutions of Maxwell's equations, corresponding to attaching a handlebody to $\mathbb{R}^{3}$. The resulting devices thus function as electromagnetic wormholes.
\end{abstract}

*AG and GU are supported by US NSF, ML by CoE-programm 213476 of Academy of Finland. 


\section{Introduction}

There has recently been considerable interest, both theoretical [16, 18, 19, 3, 13] and experimental [20], in invisibility (or "cloaking") from observation by electromagnetic (EM) waves. (See also [17] for a treatment of cloaking in the context of elasticity.) Theoretically, cloaking devices are given by specifying the conductivity $\sigma(x)$ (in the case of electrostatics), the index of refraction $n(x)$ (for optics in the absence of polarization, where one uses the Helmholtz equation), or the electric permittivity $\epsilon(x)$ and magnetic permeability $\mu(x)$ (for the full system of Maxwell's equations.) In the constructions to date, the EM parameter fields $(\sigma ; n ; \epsilon$ and $\mu$ ) have been piecewise smooth and anisotropic. (See, however, [5, Sec.4] for an example that can be interpreted as cloaking with respect to Helmholtz by an isotropic negative index of refraction material.) Furthermore, the EM parameters have singularities, with one or more eigenvalues of the tensors going to zero or infinity as one approaches from on or both sides the cloaking surface $\Sigma$, which encloses the region within which objects may be hidden from external observation. Such constructions might have remained theoretical curiosities, but the advent of metamaterials[1] allows one, within the constraints of current technology, to construct media with fairly arbitrary $\epsilon(x)$ and $\mu(x)$.

It thus becomes an interesting mathematical problem with practical significance to understand what other new phenomena of wave propagation can be produced by prescribing other arrangements of $\epsilon$ and $\mu$. Geometrically, cloaking can be viewed as arising from a singular transformation of $\mathbb{R}^{3}$. Intuitively, for a spherical cloak [6, 7, 18], it is as if an infinitesimally small hole in space has been stretched to a ball $D$; an object can be inserted inside the hole so created and is then invisible to external observations. On the level of the EM parameters, homogeneous, isotropic parameters $\epsilon, \mu$ are pushed forward to become inhomogeneous, anisotropic and singular as one approaches $\Sigma=\partial D$ from the exterior. There are then two ways, referred to as the single and double coating in [3], of continuing $\epsilon, \mu$ to within $D$ so as to rigorously obtain invisibility with respect to locally finite energy waves. We refer to either process as blowing up a point. As observed in [3], one can use the double coating to produce a manifold with a different topology, but with the change in topology invisible to external measurements.

To define the solutions of Maxwell's equations rigorously in the single coating 
case, one has to add boundary conditions on $\Sigma$. Physically, this corresponds to the lining of the interior of the single coating material, e.g., in the case of blowing up a point, with a perfectly conducting layer, see [3]. We point out here that in the recent preprint [21], the single coating construction is supplemented with selfadjoint extensions of Maxwell operators in the interior of the cloaked regions; these implicitly impose interior boundary conditions on the boundary of the cloaked region, similar to the PEC boundary condition suggested in 3. For the case of an infinite cylinder the Soft-and-Hard (SH) interior boundary condition is used in [3] to guarantee cloaking of active objects, and is needed even for passive ones.

In this paper, we show how more elaborate geometric constructions, corresponding to blowing up a curve, enable the description of tunnels which allow the passage of waves between distant points, while only the ends of the tunnels are visible to external observation. These devices function as electromagnetic wormholes, essentially changing the topology of space with respect to solutions of Maxwell's equations.

We form the wormhole device around an obstacle $K \subset \mathbb{R}^{3}$ as follows. First, one surrounds $K$ with metamaterials, corresponding to a specification of EM parameters $\widetilde{\varepsilon}$ and $\widetilde{\mu}$. Secondly, one lines the surface of $K$ with material implementing the Soft-and-Hard ( $\mathrm{SH}$ ) boundary condition from antenna theory [8, 10, 11]; this condition arose previously [3] in the context of cloaking an infinite cylinder. The EM parameters, which become singular as one approaches $K$, are given as the pushforwards of nonsingular parameters $\varepsilon$ and $\mu$ on an abstract three-manifold $M$, described in Sec. 2. For a curve $\gamma \subset M$, we construct the diffeomorphism $F$ from $M \backslash \gamma$ to the wormhole device in Sec. 3. For the resulting EM parameters $\widetilde{\varepsilon}$ and $\widetilde{\mu}$, we have singular coefficients of Maxwell's equations at $K$, and so it is necessary to formulate an appropriate notion of locally finite energy solutions (see Def. 4.1). In Theorem 4.2, we then show that there is a perfect correspondence between the external measurements of EM waves propagating through the wormhole device and those propagating on the wormhole manifold.

It was shown in [3] that the cloaking constructions are mathematically valid at all frequencies $k$. However, both cloaking and the wormhole effect studied here should be considered as essentially monochromatic, or at least very narrow-band, using current technology, since, from a practical point of view the metamaterials needed to implement the constructions have to be fabri- 
cated and assembled with a particular wavelength in mind, and theoretically are subject to significant dispersion [18]. Thus, as for cloaking in [16, 18, 3], here we describe the wormhole construction relative to electromagnetic waves at a fixed positive frequency $k$. We point out that the metamaterials used in the experimental verification of cloaking [20] should be readily adaptable to yield a physical implementation, at microwave frequencies, of the wormhole device described here. See Remark 1 in Sec. 4.2 for further discussion.

The results proved here were announced in [4].

\section{The wormhole manifold $M$}

First we explain, somewhat informally, what we mean by a wormhole. The concept of a wormhole is familiar from general relativity [9, 22], but here we define a wormhole as an object obtained by gluing together pieces of Euclidian space equipped with certain anisotropic EM parameter fields. We start by describing this process heuristically; later, we explain more precisely how this can be effectively realized vis-a-vis EM wave propagation using metamaterials.

We first describe the wormhole as an abstract manifold $M$, see Fig. 1 ; in the next section we will show how to realize this concretely in $\mathbb{R}^{3}$, as a wormhole device $N$. Start by making two holes in the Euclidian space $\mathbb{R}^{3}=$ $\{(x, y, z) \mid x, y, z \in \mathbb{R}\}$, say by removing the open ball $B_{1}=B(O, 1)$ with center at the origin $O$ and of radius 1 , and also the open ball $B_{2}=B(P, 1)$, where $P=(0,0, L)$ is a point on the $z$-axis having the distance $L>3$ to the origin. We denote by $M_{1}$ the region so obtained, $M_{1}=\mathbb{R}^{3} \backslash\left(B_{1} \cup B_{2}\right)$, which is the first component we need to construct a wormhole. Note that $M_{1}$ is a 3-dimensional manifold with boundary, the boundary of $M_{1}$ being $\partial M_{1}=\partial B_{1} \cup \partial B_{2}$, the union of two 2-spheres. Thus, $\partial M_{1}$ can be considered as a disjoint union $\mathbb{S}^{2} \cup \mathbb{S}^{2}$, where we will use $\mathbb{S}^{2}$ to denote various copies of the two-dimensional unit sphere.

The second component needed is a 3-dimensional cylinder, $M_{2}=\mathbb{S}^{2} \times[0,1]$. This cylinder can be constructed by taking the closed unit cube $[0,1]^{3}$ in $\mathbb{R}^{3}$ and, for each value of $0<s<1$, gluing together, i.e., identifying, all of the points on the boundary of the cube with $z=s$. Note that we do not identify points at the top of the boundary, at $z=1$, or at the bottom, at $z=0$. We 
then glue together the boundary $\partial B(O, 1) \sim \mathbb{S}^{2}$ of the ball $B(O, 1)$ with the lower end (boundary component) $\mathbb{S}^{2} \times\{0\}$ of $M_{2}$, and the boundary $\partial B(P, 1)$ with the upper end, $\mathbb{S}^{2} \times\{1\}$. In doing so we identify the point $(0,0,1) \in$ $\partial B(O, 1)$ with the point $N P \times\{0\}$ and the point $(0,0, L-1) \in \partial B(P, 1)$ with the point $N P \times\{1\}$, where $N P$ is the north pole on $\mathbb{S}^{2}$.

The resulting manifold $M$ no longer lies in $\mathbb{R}^{3}$, but rather is the connected sum of the components $M_{1}$ and $M_{2}$ and has the topology of $\mathbb{R}^{3}$ with a 3-dimensional handle attached. Note that adding this handle makes it possible to travel from one point in $M_{1}$ to another point in $M_{1}$, not only along curves lying in $M_{1}$ but also those in $M_{2}$.

To consider Maxwell's equations on $M$, let us start with Maxwell's equations on $\mathbb{R}^{3}$ at frequency $k \in \mathbb{R}$, given by

$\nabla \times E=i k B, \quad \nabla \times H=-i k D, \quad D(x)=\varepsilon(x) E(x), \quad B(x)=\mu(x) H(x)$.

Here $E$ and $H$ are the electric and magnetic fields, $D$ and $B$ are the electric displacement field and the magnetic flux density, $\varepsilon$ and $\mu$ are matrices corresponding to permittivity and permeability. As the wormhole is topologically different from the Euclidian space $\mathbb{R}^{3}$, we use a formulation of Maxwell's equations on a manifold, and as in [3], do this in the setting of a general Riemannian manifold, $(M, g)$. For our purposes, as in [14, 3] it suffices to use $\varepsilon, \mu$ which are conformal, i.e., proportional by scalar fields, to the metric $g$. In this case, Maxwell's equations can be written, in the coordinate invariant form, as

$$
d E=i k B, d H=-i k D, \quad D=\epsilon E, B=\mu H \quad \text { in } M,
$$

where $E, H$ are 1 -forms, $D, B$ are 2-forms, $d$ is the exterior derivative, and $\epsilon$ and $\mu$ are scalar functions times the Hodge operator of $(M, g)$, which maps 1 -forms to the corresponding 2-forms [2]. In local coordinates these equations are written in the same form as Maxwell's equations in Euclidian space with matrix valued $\varepsilon$ and $\mu$. Although not necessary, for simplicity one can choose a metric on the wormhole manifold $M$ which is Euclidian on $M_{1}$, and on $M_{2}$ is the product of a given metric $g_{0}$ on $\mathbb{S}^{2}$ and the standard metric of $[0,1]$. More generally, can also choose the metric on $M_{2}$ to be a warped product. Even the simple choice of the product of the standard metric of $\mathbb{S}^{2}$ and the metric $\delta^{2} d s^{2}$, where $\delta$ is the "length" of the wormhole, gives rise to interesting ray-tracing effects for rays passing through the wormhole tunnel. For $\delta<<1$, 
the image through one end of the wormhole (of the region beyond the other end) would resemble the image in a a fisheye lens; for $\delta \gtrsim 1$, multiple images and greater distortion occur. (See [4, Fig.2].)

The proof of the wormhole effect that we actually give is for yet another variation, where the balls that form the ends have their boundary spheres flattened; this may be useful for applications, since it allows for there to be a vacuum (or air) in a neighborhood of the axis of the wormhole, so that, e.g., instruments may be passed through the wormhole. We next show how to construct, using metamaterials, a device $N$ in $\mathbb{R}^{3}$ that effectively realizes the geometry and topology of $M$, relative to solutions of Maxwell's equations at frequency $k$, and hence functions as an electromagnetic wormhole.

\section{The wormhole device $N$ in $\mathbb{R}^{3}$}

We now explain how to construct a "device" $N$ in $\mathbb{R}^{3}$, i.e., a specification of permittivity $\varepsilon$ and permeability $\mu$, which affects the propagation of electromagnetic waves in the same way as the presence of the handle $M_{2}$ in the wormhole manifold $M$. What this means is that we prescribe a configuration of metamaterials which make the waves behave as if there were an invisible tube attached to $\mathbb{R}^{3}$, analogous to the handle $M_{2}$ in the wormhole manifold $M$. In the other words, as far as external EM observations of the wormhole device are concerned, it appears as if the topology of space has been changed.

We use cylindrical coordinates $(\theta, r, z)$ corresponding to a point $(r \cos \theta, r \sin \theta, z)$ in $\mathbb{R}^{3}$. The wormhole device is built around an obstacle $K \subset \mathbb{R}^{3}$. To define $K$, let $S$ be the two-dimensional finite cylinder $\{\theta \in[0,2 \pi], r=2,0 \leq$ $z \leq L\} \subset \mathbb{R}^{3}$. The open region $K$ consists of all points in $\mathbb{R}^{3}$ that have distance less than one to $S$ and has the shape of a long, thick-walled tube with smoothed corners.

Let us first introduce a deformation map $F$ from $M$ to $N=\mathbb{R}^{3} \backslash K$ or, more precisely, from $M \backslash \gamma$ to $N \backslash \Sigma$, where $\gamma$ is a closed curve in $M$ to be described shortly and $\Sigma=\partial K$. We will define $F$ separately on $M_{1}$ and $M_{2}$ denoting the corresponding parts by $F_{1}$ and $F_{2}$.

To describe $F_{1}$, let $\gamma_{1}$ be the line segment on the $z$-axis connecting $\partial B(O, 1)$ and $\partial B(P, 1)$ in $M_{1}$, namely, $\gamma_{1}=\{r=0, z \in[1, L-1]\}$. Let $F_{1}(r, z)=$ $(\theta, R(r, z), Z(r, z))$ be such that $(R(r, z), Z(r, z))$, shown in Fig. 2, 

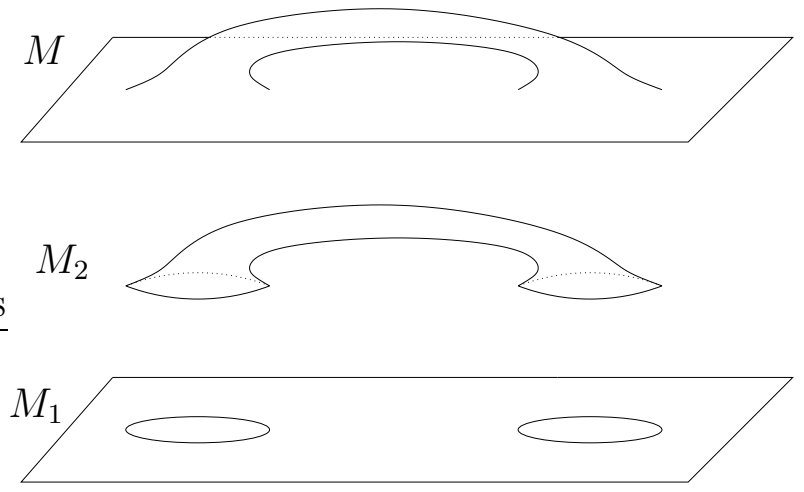

Figure 1: Schematic figure: a wormhole manifold is glued from two components, the "handle" and space with two holes. Note that in the actual construction, the components are three dimensional.
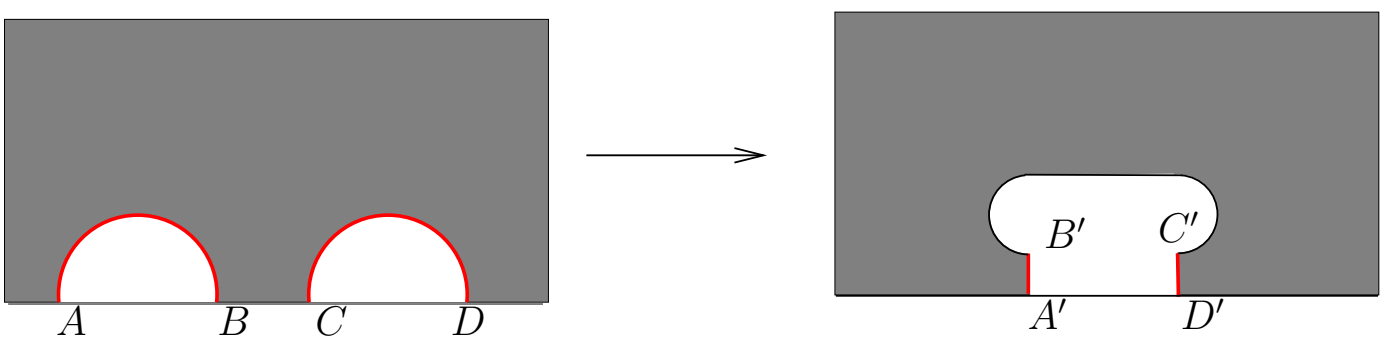

Figure 2: The map $(R(r, z), Z(r, z))$ in cylindrical coordinates $(z, r)$. 
transforms in the $(r, z)$ coordinates the semicircles $A B$ and $C D$ in the left picture to the vertical line segments $A^{\prime} B^{\prime}=\{r \in[0,1], z=0\}$ and $C^{\prime} D^{\prime}=$ $\{r \in[0,1], z=L\}$ in the right picture and the cut $\gamma_{1}$ on the left picture to the curve $B^{\prime} C^{\prime}$ on the right picture. This gives us a map $F_{1}: M_{1} \backslash \gamma_{1} \rightarrow N_{1} \backslash \Sigma$, where the closed region $N_{1}$ in $\mathbb{R}^{3}$ is obtained by rotation of the region exterior to the curve $A^{\prime} B^{\prime} C^{\prime} D^{\prime}$ around the $z$-axis. We can choose $F_{1}$ so that it is the identity map in the domain $U=\mathbb{R}^{3} \backslash\{-2 \leq z \leq L+2,0 \leq r \leq 4\}$.

To describe $F_{2}$, consider the line segment, $\gamma_{2}=\{N P\} \times[0,1]$ on $M_{2}$. The sphere without the north pole can be "flattened" and stretched to an open disc with radius one which, together with stretching $[0,1]$ to $[0, L]$, gives us a map $F_{2}$ from $M_{2} \backslash \gamma_{2}$ to $N_{2} \backslash \Sigma$. The region $N_{2}$ is the 3 -dimensional cylinder, $N_{2}=\{\theta \in[0,2 \pi], r \in[0,1], z \in[0, L]\}$. When flattening $\mathbb{S}^{2} \backslash N P$, we do it in such a way that $F_{1}$ on $\partial B(O, 1)$ and $\partial B(P, 1)$ coincides with $F_{2}$ on $\left(\mathbb{S}^{2} \backslash N P\right) \times\{0\}$ and $\left(\mathbb{S}^{2} \backslash N P\right) \times\{1\}$, respectively.

Thus, $F$ maps $M \backslash \gamma$, where $\gamma=\gamma_{1} \cup \gamma_{2}$ is a closed curve in $M$, onto $N \backslash \Sigma$; in addition, $F$ is the identity on the region $U$.

Now we are ready to define the electromagnetic material parameter tensors on $N$. We define the permittivity to be

$$
\widetilde{\varepsilon}=F_{*} \varepsilon(y)=\left.\frac{(D F)(x) \cdot \varepsilon(x) \cdot(D F(x))^{t}}{\operatorname{det}(D F)}\right|_{x=F^{-1}(y)},
$$

where $D F$ is the derivative matrix of $F$, and similarly the permeability to be $\widetilde{\mu}=F_{*} \mu$. These deformation rules are based on the fact that permittivity and permeability are conductivity type tensors, see [14].

Maxwell's equations are invariant under smooth changes of coordinates. This means that, by the chain rule, any solution to Maxwell's equations in $M \backslash \gamma$, endowed with material parameters $\varepsilon, \mu$ becomes, after transformation by $F$, a solution to Maxwell's equations in $N \backslash \Sigma$ with material parameters $\widetilde{\varepsilon}$ and $\widetilde{\mu}$, and vice versa. However, when considering the fields on the entire spaces $M$ and $N$, these observations are not enough, due to the singularities of $\widetilde{\varepsilon}$ and $\widetilde{\mu}$ near $\Sigma$; the significance of this for cloaking was observed and analyzed in [3]. In the following, we will show that the physically relevant class of solutions to Maxwell's equations, namely the (locally) finite energy solutions, remains the same, with respect to the transformation $F$, in $(M ; \varepsilon, \mu)$ and $(N ; \widetilde{\varepsilon}, \widetilde{\mu})$. One can analyze the rays in $M$ and $N$ endowed with the electromagnetic wave propagation metrics $g=\sqrt{\varepsilon \mu}$ and $\widetilde{g}=\sqrt{\widetilde{\varepsilon \mu}}$, respectively. Then the rays on 
$M$ are transformed by $F$ into the rays in $N$. As almost all the rays on $M$ do not intersect with $\gamma$, therefore, almost all the rays on $N$ do not approach $\Sigma$. This was the basis for [16, 18] and was analyzed further in [19]; see also [17] for a similar analysis in the context of elasticity. Thus, heuristically one is led to conclude that the electromagnetic waves on $(M ; \varepsilon, \mu)$ do not feel the presence of $\gamma$, while those on $(N ; \widetilde{\varepsilon}, \widetilde{\mu})$ do not feel the presence of $K$, and these waves can be transformed into each other by the map $F$.

Although the above considerations are mathematically rigorous, on the level both of the chain rule and of high frequency limits, i.e., ray tracing, in the exteriors $M \backslash \gamma$ and $N \backslash \Sigma$, they do not suffice to fully describe the behavior of physically meaningful solution fields on $M$ and $N$. However, by carefully examining the class of the finite-energy waves in $M$ and $N$ and analyzing their behavior near $\gamma$ and $\Sigma$, respectively, we can give a complete analysis, justifying the conclusions above. Let us briefly explain the main steps of the analysis using methods developed for theory of invisibility (or cloaking) at frequency $k>0$ [3] and at frequency $k=0$ in [6, 7]. The details will follow.

First, to guarantee that the fields in $N$ are finite energy solutions and do not blow up near $\Sigma$, we have to impose at $\Sigma$ the appropriate boundary condition, namely, the Soft-and-Hard (SH) condition, see [8, 11],

$$
\left.e_{\theta} \cdot E\right|_{\Sigma}=0,\left.\quad e_{\theta} \cdot H\right|_{\Sigma}=0,
$$

where $e_{\theta}$ is the angular direction. Secondly, the map $F$ can be considered as a smooth coordinate transformation on $M \backslash \gamma$; thus, the finite energy solutions on $M \backslash \gamma$ transform under $F$ into the finite energy solutions on $N \backslash \Sigma$, and vice versa. Thirdly, the curve $\gamma$ in $M$ has Hausdorff dimension equal to one. This implies that the possible singularities of the finite energy electromagnetic fields near $\gamma$ are removable [12], that is, the finite energy fields in $M \backslash \gamma$ are exactly the restriction to $M \backslash \gamma$ of the fields defined on all of $M$.

Combining these steps we can see that measurements of the electromagnetic fields on $(M ; \varepsilon, \mu)$ and on $\left(\mathbb{R}^{3} \backslash K ; \widetilde{\varepsilon}, \widetilde{\mu}\right)$ coincide in $U$. In the other words, if we apply any current on $U$ and measure the radiating electromagnetic fields it generates, then the fields on $U$ in the wormhole manifold $(M ; \varepsilon, \mu)$ coincide with the fields on $U$ in $\left(\mathbb{R}^{3} \backslash K ; \widetilde{\varepsilon}, \widetilde{\mu}\right)$, 3-dimensional space equipped with the wormhole device construction.

Summarizing our construction, the wormhole device consists of the metama- 
terial coating of the obstacle $K$. This coating should have the permittivity $\widetilde{\varepsilon}$ and permeability $\widetilde{\mu}$. In addition, we need to impose the SH boundary condition on $\Sigma$, which may be realized by fabricating the obstacle $K$ from a perfectly conducting material with parallel corrugations on its surface [8, 11].

In the next section, the permittivity $\widetilde{\varepsilon}$ and and permeability $\widetilde{\mu}$ are described in a rather simple form. (As mentioned earlier, in order to allow for a tube around the axis of the wormhole to be a vacuum or air, we deal with a slightly different construction than was described above, starting with flattened spheres). It should be possible to physically implement an approximation to this mathematical idealization of the material parameters needed for the wormhole device, using concentric rings of split ring resonators as in the experimental verification of cloaking obtained in [20].

\section{Rigorous construction of the wormhole}

Here we present a rigorous model of a typical wormhole device and justify the claims above concerning the behavior of the electromagnetic fields in the wormhole device in $\mathbb{R}^{3}$ in terms of as the fields on the wormhole manifold $(M, g)$.

\subsection{The wormhole manifold $(M, g)$ and the wormhole device $N$}

Here we prove the wormhole effect for a variant of the wormhole device described in the previous sections. Instead of using a round sphere $\mathbb{S}^{2}$ as before, we present a construction that uses a deformed sphere $\mathbb{S}_{\text {flat }}^{2}$ that is flat the near the south and north poles, $S P$ and $N P$. This makes it possible to have constant isotropic material parameters near the $z$-axis located inside the wormhole. For possible applications, see [4].

We use following notations. Let $(\theta, r, z) \in[0,2 \pi] \times \overline{\mathbb{R}}_{+} \times \mathbb{R}$ be the cylindrical coordinates of $\mathbb{R}^{3}$, that is the map

$$
X:(\theta, r, z) \rightarrow(r \cos \theta, r \sin \theta, z)
$$

that maps $X:[0,2 \pi] \times \overline{\mathbb{R}}_{+} \times \mathbb{R} \rightarrow \mathbb{R}^{3}$. In the following, we identify $[0,2 \pi]$ and the unit circle $S^{1}$. 
Let us start by removing from $\mathbb{R}^{3}$ two "deformed" balls which have flat portions near the south and north poles. More precisely, let $M_{1}=\mathbb{R}^{3} \backslash\left(P_{1} \cup\right.$ $P_{2}$ ), where in the cylindrical coordinates

$$
\begin{aligned}
P_{1}= & \{X(\theta, r, z):-1 \leq z \leq 1,0 \leq r \leq 1\} \\
& \cup\left\{X(\theta, r, z):(r-1)^{2}+z^{2} \leq 1\right\}, \\
P_{2}=\{ & X(\theta, r, z): 1 \leq z-L \leq 1,0 \leq r \leq 1\} \\
& \cup\left\{X(\theta, r, z):(r-1)^{2}+(z-L)^{2} \leq 1\right\} .
\end{aligned}
$$

We say that the boundary $\partial P_{1}$ of $P_{1}$ is a deformed sphere with flat portions, and denote it by $\mathbb{S}_{\text {flat }}^{2}$. We say that the intersection points of $\mathbb{S}_{\text {flat }}^{2}$ with the $z$-axis are the north pole, $N P$, and the south pole, $S P$.

Let $g_{1}$ be the metric on $M_{1}$ inherited from $\mathbb{R}^{3}$, and let $\gamma_{1}$ be the path

$$
\gamma_{1}=\{X(0,0, z): 1<z<L-1\} \subset M_{1} .
$$

Set

$$
\begin{aligned}
A_{1} & =M_{1} \backslash V_{1 / 4}, \\
V_{t} & =\{X(\theta, r, z): 0 \leq r \leq t, 1<z<L-1\}, \quad 0<t<1,
\end{aligned}
$$

and consider a map $G_{0}: M_{1} \backslash \gamma_{1} \rightarrow A_{1}$; see Fig. 3. $G_{0}$ defined as the identity map on $M_{1} \backslash V_{1 / 2}$ and, in cylindrical coordinates, as

$$
G_{0}(X(\theta, r, z))=X\left(\theta, \frac{1}{4}+\frac{r}{2}, z\right), \quad(\theta, r, z) \in V_{1 / 2} .
$$

Clearly, $G_{0}$ is $C^{0,1}$-smooth.

Let $U(x) \in \mathbb{R}^{3 \times 3}, x=X(\theta, r, z)$, be the orthogonal matrix that maps the standard unit vectors $e_{1}, e_{2}, e_{3}$ of $\mathbb{R}^{3}$ to the Euclidian unit vectors corresponding to the $\theta, r$, and $z$ directions, that is,

$U(x) e_{1}=(-\sin \theta, \cos \theta, 0), \quad U(x) e_{2}=(\cos \theta, \sin \theta, 0), \quad U(x) e_{3}=(0,0,1)$.

Then the differential of $G_{0}$ in the Euclidian coordinates at the point $x \in V_{1 / 2}$ is the matrix

$$
D G_{0}(x) U(y)\left(\begin{array}{ccc}
\frac{1}{r}\left(\frac{1}{4}+\frac{r}{2}\right) & 0 & 0 \\
0 & \frac{1}{2} & 0 \\
0 & 0 & 1
\end{array}\right) U(x)^{-1}, \quad x=X(\theta, r, z), y=G_{0}(x) .
$$


Later we impose on part of the boundary, $\Sigma_{0}=\partial A_{1} \cap\{1<z<L-1\}$, the soft-and-hard boundary condition (marked red in the figures).

Next, let $(\theta, z, \tau)=(\theta(x), z(x), \tau(x))$ be the Euclidian boundary normal coordinates associated to $\Sigma_{0}$, that is, $\tau(x)=\operatorname{dist}_{\mathbb{R}^{3}}\left(x, \Sigma_{0}\right)$ and $(\theta(x), z(x))$ are the $\theta$ and $z$-coordinates of the closest point of $\Sigma_{0}$ to $x$.

Denote by $\left(G_{0}\right)_{*} g_{1}$ the push forward of the metric $g_{1}$ in $G_{0}$, that is, the metric obtained from $g_{1}$ using the change of coordinates $G_{0}$, see [2]. The metric $\left(G_{0}\right)_{*} g_{1}$ coincides with $g_{1}$ in $A_{1} \backslash V_{1 / 2}$, and in the Euclidian boundary normal coordinates of $\Sigma_{0}$, on $A_{1} \cap V_{1 / 2}$, the metric $\left(G_{0}\right)_{*} g_{1}$, has the length element

$$
d s^{2}=4 \tau^{2} d \theta^{2}+d z^{2}+4 d \tau^{2}
$$
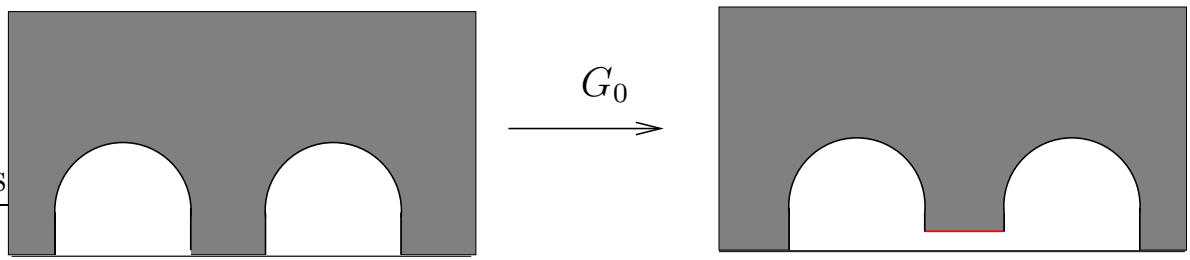

Figure 3: A schematic figure on the map $G_{0}$, considered in the $(r, z)$ coordinates. Later, we impose the $\mathrm{SH}$ boundary condition on the portion of the boundary coloured red.

Next, let

$$
\begin{aligned}
& q_{3}= \operatorname{conv}\left(\left\{(r, z):(r-2)^{2}+(z-(-2))^{2} \leq 1\right\}\right. \\
&\left.\cup\left\{(r, z):(r-2)^{2}+(z-(L+2))^{2} \leq 1\right\}\right), \\
& q_{4}=\{(r, z): 0 \leq r \leq 1,-1 \leq z \leq L+1\},
\end{aligned}
$$

where $\operatorname{conv}(q)$ denotes the convex hull of the set $q$.

Let

$$
\begin{aligned}
N_{1} & =\mathbb{R}^{3} \backslash\left(P_{3} \cup P_{4}\right), \\
P_{3} & =\left\{X(\theta, r, z):(r, z) \in q_{3}\right\}, \\
P_{4} & =\left\{X(\theta, r, z):(r, z) \in q_{4}\right\}, \\
\Sigma_{1} & =\partial N_{1} \backslash \partial P_{4} .
\end{aligned}
$$


We can find a Lipschitz smooth map $G_{1}: A_{1} \rightarrow N_{1}$, see Fig. 4, of the form

$$
G_{1}(X(\theta, r, z))=X(\theta, R(r, z), Z(r, z))
$$

such that it maps $\Sigma_{0}$ to $\Sigma_{1}$, and in $A_{1}$ near $\Sigma_{0}$ it is given by

$$
G_{1}\left(x+t \nu_{0}\right)=G_{1}(x)+t \nu_{1}
$$

Here, $x \in \Sigma_{0}, \nu_{0}$ is the Euclidian unit normal vector of $\Sigma_{0}, \nu_{1}$ is the Euclidian unit normal vector of $\Sigma_{1}$, and $0<t<\frac{1}{4}$. Moreover, we can find a $G_{1}$ so that it is the identity map near the $z$-axis, that is,

$$
G_{1}(x)=x, \quad x \in A_{1} \cap\left\{0 \leq r<\frac{1}{4}\right\}
$$

and such that $G_{1}$ is also the identity map in the set of points with the Euclidian distance 4 or more from $P_{1} \cup P_{2}$. Note that we can find such a $G_{1}$ such that both $G_{1}$ and its inverse $G_{1}^{-1}$ are Lipschitz smooth up to the boundary. Thus the differential $D G_{1}$ of $G_{1}$ at $x \in A_{1}$ in Euclidian coordinates is

$D G_{1}(x)=U(y)\left(\begin{array}{cc}a_{11}(r, z) & 0 \\ 0 & A(r, z)\end{array}\right) U(x)^{-1}, \quad x=X(\theta, r, z), y=G_{1}(x)$,

where $c_{0} \leq a_{11}(r, z) \leq c_{1}$ and $A(r, z)$ is a symmetric $(2 \times 2)$-matrix satisfying

$$
c_{0} I \leq A(r, z) \leq c_{1} I
$$

with some $c_{0}, c_{1}>0$.

The map $F_{1}(x)=G_{1}\left(G_{0}(x)\right)$ then maps $F_{1}: M_{1} \backslash \gamma_{1} \rightarrow N_{1}$. Let $\widetilde{g}_{1}=\left(F_{1}\right)_{*} g_{1}$ be metric on $N_{1}$. From the above considerations, we see that the differential $D F_{1}$ of $F_{1}$ at $x \in M_{1} \backslash \gamma_{1}$ near $\Sigma_{0}$, in Euclidian coordinates, is given by

$$
\begin{aligned}
& D F_{1}(x)=U(y)\left(\begin{array}{cc}
b_{11}(\theta, r, z) & 0 \\
0 & B(r, z)
\end{array}\right) U(x)^{-1} \\
& b_{11}(\theta, r, z)=\frac{c_{11}(r, z)}{\operatorname{dist}_{\mathbb{R}^{3}}\left(X(\theta, r, z), \Sigma_{0}\right)} \quad x=X(\theta, r, z), y=F_{1}(x)
\end{aligned}
$$

where $c_{0} \leq c_{11}(r, z) \leq c_{1}$, and $B(r, z)$ is a symmetric $(2 \times 2)$-matrix satisfying

$$
c_{0} I \leq B(r, z) \leq c_{1} I,
$$


for some $c_{0}, c_{1}>0$.

Note that $\partial P_{4} \cap\{r<1\}$ consists of two two-dimensional discs, $B_{2}(0,1) \times\{-1\}$ and $B_{2}(0,1) \times\{L+1\}$. Below, we will use the map

$$
f_{2}=\left.F_{1}\right|_{\partial P_{1} \backslash N P}: \partial P_{1} \backslash N P \rightarrow B_{2}(0,1) \times\{-1\} \subset \partial N_{1} .
$$

The map $f_{2}$ can be considered as the deformation that "flattens" $\mathbb{S}_{\text {flat }}^{2} \backslash N P$ to a two dimensional unit disc.
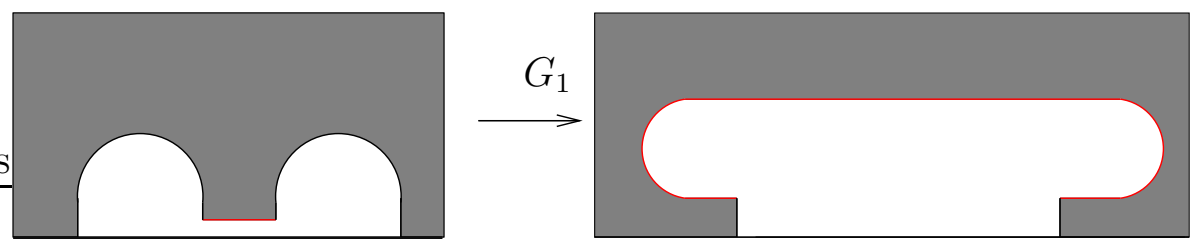

Figure 4: Map $G_{1}$ in $(r, z)$-coordinates.

To describe $f_{2}$, consider $\mathbb{S}_{\text {flat }}^{2}$ as a surface in Euclidian space and define on it the $\theta$ coordinate corresponding to the $\theta$ coordinate of $\mathbb{R}^{3} \backslash\{z=0\}$. Let then $s(y)$ be the intrinsic distance of $y \in \mathbb{S}_{\text {flat }}^{2}$ to the south pole $S P$. Then $(\theta, s)$ define coordinates in $\mathbb{S}_{\text {flat }}^{2} \backslash\{S P, N P\}$. We denote by $y(\theta, s) \in \mathbb{S}_{\text {flat }}^{2} \backslash\{S P, N P\}$ the point corresponding to the coordinates $(\theta, s)$.

By the above construction, the map $f_{2}$ has the form, with respect to the coordinates used above,

$$
\begin{aligned}
& f_{2}(y(\theta, s))=X(\theta, R(s),-1) \in B_{2}(0,1) \times\{-1\}, \quad \text { where } \\
& R(s)=s, \quad \text { for } 0<s<\frac{1}{4}, \\
& R(s)=1-\frac{1}{2}[(\pi+4)-s], \quad \text { for }(\pi+4)-\frac{1}{4}<s<(\pi+4),
\end{aligned}
$$

cf. formulae (2) and (3). In the following we identify $B_{2}(0,1) \times\{-1\}$ with the disc $B_{2}(0,1)$.

Let $h_{1}$ be the metric on $\partial P_{1} \backslash N P$ inherited from $\left(M_{1}, g_{1}\right)$. Let $h_{2}=\left(f_{2}\right)_{*} h_{1}$ be the metric on $B_{2}(0,1)$. We observe that the metric $h_{2}$ makes the disc $B_{2}(0,1)$ isometric to $\mathbb{S}_{\text {flat }}^{2} \backslash N P$, endowed with the metric inherited from $\mathbb{R}^{3}$. Thus, let

$$
M_{2}=\mathbb{S}_{\text {flat }}^{2} \times[-1, L+1]
$$


On $M_{2}$, let the metric $g_{2}$ be the product of the metric of $\mathbb{S}_{\text {flat }}^{2}$ inherited from $\mathbb{R}^{3}$ and the metric $\alpha_{2}(z) d z^{2}, \alpha_{2}>0$ on $[-1, L+1]$. Let $\gamma_{2}=\{N P\} \times[-1, L+1]$ be a path on $M_{2}$.

Define $N_{2}=P_{4}=\{X(\theta, r, z): 0 \leq r<1,-1 \leq z \leq L+1\} \subset \mathbb{R}^{3}$, $\Sigma_{2}=\partial N_{2} \cap\{r=1\}$, and let $F_{2}: M_{2} \backslash \gamma_{2} \rightarrow N_{2}$ be the map of the form

$$
F_{2}(y, z)=\left(f_{2}(y), z\right) \in \mathbb{R}^{3}, \quad(y, z) \in\left(\mathbb{S}_{\text {flat }}^{2} \backslash N P\right) \times[-1, L+1] .
$$

Let $\widetilde{g}_{2}=\left(F_{2}\right)_{*} g_{2}$ be the resulting metric on $N_{2}$.

Figure 5: The set $N_{2}$ in the $(r, z)$ coordinates. Later, we impose the SH boundary condition on the portion of the boundary colored red.

Denote by $\bar{M}_{1}=M_{1} \cup \partial M_{1}$ the closure of $M_{1}$ and let $(M, g)=\left(\bar{M}_{1}, g_{1}\right) \#\left(M_{2}, g_{2}\right)$ be the connected sum of $\bar{M}_{1}$ and $M_{2}$, that is, we glue the boundaries $\partial M_{1}$ and $\partial M_{2}$. The set $N=N_{1} \cup N_{2} \subset \mathbb{R}^{3}$ is open, and its boundary $\partial N$ is $\Sigma=\Sigma_{1} \cup \Sigma_{2}$.

Let $F$ be the map $F: M \backslash \gamma \rightarrow N$ defined by the maps $F_{1}: M_{1} \backslash \gamma_{1} \rightarrow N_{1}$ and $F_{2}: M_{2} \backslash \gamma_{2} \rightarrow N_{2}$, and finally, let $\gamma=\gamma_{1} \cup \gamma_{2}$ and $\widetilde{g}=F_{*} g$.

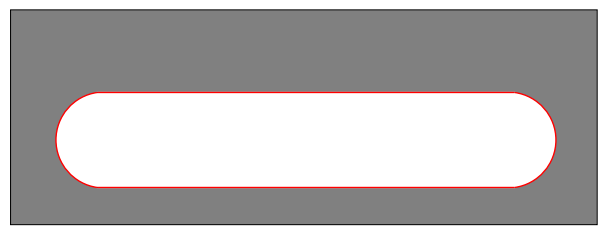

Figure 6: The set $N=N_{1} \cup N_{2} \subset \mathbb{R}^{3}$ having the complement $K$, presented in the $(r, z)$ coordinates. Later, the $\mathrm{SH}$ boundary condition is imposed on $\partial K$.

Let $K=\mathbb{R}^{3} \backslash N$. On the surface $\Sigma=\partial K$ we can use local coordinates $(\widetilde{t}, \widetilde{\theta})$, where $\tilde{\theta}$ is the $\theta$-coordinate of the ambient space $\mathbb{R}^{3}$ and $\widetilde{t}$ is either the $r$ or 
$z$-coordinate of the ambient space $\mathbb{R}^{3}$ restricted to $\Sigma$. Denote also

$$
\widetilde{\tau}=\widetilde{\tau}(x)=\operatorname{dist}_{\mathbb{R}^{3}}(x, \partial K) .
$$

Then by formula (21) we see that in $N_{1}$, in the Euclidian boundary normal coordinates $(\widetilde{\theta}, \widetilde{t}, \widetilde{\tau})$ associated to the surface $\Sigma_{1}$, the metric $\widetilde{g}$ has the length element

$d s^{2}=4 d \widetilde{\tau}^{2}+\alpha_{1}(\widetilde{t}) d \widetilde{t}^{2}+4 \widetilde{\tau}^{2} d \widetilde{\theta}^{2}, \quad 0<\widetilde{\tau}<\frac{1}{4}, \quad c_{0}^{-1} \leq \alpha_{1}(\widetilde{t}) \leq c_{0}, c_{0} \geq 1$.

The construction of $F_{2}$ yields that in $N_{2}$, in the Euclidian boundary normal coordinates $(\widetilde{\theta}, \widetilde{t}, \widetilde{\tau})$ with $\widetilde{t}=z$, associated to the surface $\Sigma_{2}=\partial K \cap \partial N_{2}$, the metric $\widetilde{g}$ has the length element, near $\Sigma_{2}$,

$$
d s^{2}=4 d \widetilde{\tau}^{2}+\alpha_{2}(\widetilde{t}) d \widetilde{t}^{2}+4 \widetilde{\tau}^{2} d \widetilde{\theta}^{2}, \quad 0<\widetilde{\tau}<\frac{1}{4} .
$$

Here, near $\partial N_{1} \cap \partial N_{2}$, we use $\widetilde{t}=z$ on $\Sigma_{1}$. Choosing the map $G_{1}$ in the construction of the map $F_{1}$ appropriately, we have $\alpha_{2}(-1)=\alpha_{1}(-1), \alpha_{2}(L+$ $1)=\alpha_{1}(L+1)$, and the resulting map is Lipschitz.

On $M_{1}, N_{1}$, and $N_{2}$ that are subsets of $\mathbb{R}^{3}$ we have the well defined cylindrical coordinates $(\theta, r, z)$. Similarly, $M_{2}=\mathbb{S}_{\text {flat }}^{2} \times[-1, L+1]$ we define the coordinates $(\theta, s, z)$, where $(\theta, s)$ are the above defined coordinates on $\mathbb{S}_{\text {flat }}^{2} \backslash\{S P, N P\}$.

We can also consider on $N \subset \mathbb{R}^{3}$ also the Euclidian metric, denoted by $g^{e}$. In Euclidean coordinates, $\left(g^{e}\right)_{i j}=\delta_{j k}$. Consider next the above defined Euclidian boundary normal coordinates $(\widetilde{\theta}, \widetilde{t}, \widetilde{\tau})$ associated to $\partial K$. They are well defined in a neighborhood of $\partial K$. We define the vector fields

$$
\widetilde{\xi}=\partial_{\widetilde{\tau}}, \quad \widetilde{\eta}=\partial_{\widetilde{\theta}}, \quad \widetilde{\zeta}=\partial_{\tilde{t}}
$$

on $N$ near $\partial K$. These vector fields are orthogonal with respect to the metric $\widetilde{g}$ and to the metric $g^{e}$.

On $M$ near $\gamma$, we use coordinates $(\theta, t, \tau)$. On $M_{1}$, near $\gamma_{1}$ they in the terms of the cylindrical coordinates are $(\theta, t, \tau)=(\theta, z, r)$. On $M_{2}$, they are the coordinates $(\theta, t, \tau)=(\theta, z, s)$, where $s$ is the intrinsic distance to the north pole $N P$. We define also the vector fields

$$
\xi=\partial_{\tau}, \quad \eta=\partial_{\theta}, \quad \zeta=\partial_{t}
$$


on $M \backslash \gamma$ near $\gamma$. These vector fields are orthogonal with respect to the metric $g$.

In the sequel, we consider the differential of $F$ as the linear map $D F:\left(T_{x} M, g\right) \rightarrow\left(T_{y} N, g^{e}\right), y=F(x), x \in M \backslash \gamma$.

Using formula (4) in $M_{1}$ and formulas (5), (6) in $M_{2}$, we see that $D F^{-1}(x)$ at $x \in N$ near $\partial N$ is a bounded linear map that satisfies

$$
\begin{array}{rll}
\left|\left(\eta, D F^{-1}(x) \widetilde{\eta}\right)_{g}\right| \leq C \widetilde{\tau}(x), & \left(\zeta, D F^{-1}(x) \widetilde{\eta}\right)_{g}=0, & \left(\xi, D F^{-1}(x) \widetilde{\eta}\right)_{g}=0, \\
\left(\eta, D F^{-1}(x) \widetilde{\zeta}\right)_{g}=0, & \left|\left(\zeta, D F^{-1}(x) \widetilde{\zeta}\right)_{g}\right| \leq C, & \left|\left(\xi, D F^{-1}(x) \widetilde{\zeta}\right)_{g}\right| \leq C, \\
\left(\eta, D F^{-1}(x) \widetilde{\xi}\right)_{g}=0, & \left|\left(\zeta, D F^{-1}(x) \widetilde{\xi}\right)_{g}\right| \leq C, & \left|\left(\xi, D F^{-1}(x) \widetilde{\xi}\right)_{g}\right| \leq C,
\end{array}
$$

where $C>0$ and $(\cdot, \cdot)_{g}$ is the inner product defined by the metric $g$. Moreover, we obtain similar estimates for $D F$ in terms of the Euclidian metric $g^{e}$,

$$
\begin{array}{rll}
\left|(\widetilde{\eta}, D F(y) \eta)_{g^{e}}\right| \leq C \tau(y)^{-1}, & (\widetilde{\zeta}, D F(y) \eta)_{g^{e}}=0, & (\widetilde{\xi}, D F(y) \eta)_{g^{e}}=0, \\
(\widetilde{\eta}, D F(y) \zeta)_{g^{e}}=0, & \left|(\widetilde{\zeta}, D F(y) \zeta)_{g^{e}}\right| \leq C, & \left|(\widetilde{\xi}, D F(y) \zeta)_{g^{e}}\right| \leq C, \\
(\widetilde{\eta}, D F(y) \xi)_{g^{e}}=0, & \left|(\widetilde{\zeta}, D F(y) \xi)_{g^{e}}\right| \leq C, & \left|(\widetilde{\xi}, D F(y) \xi)_{g^{e}}\right| \leq C
\end{array}
$$

for $y \in M \backslash \gamma$ near $\gamma$ with $C>0$.

Next, consider $D F(y)$ at $y \in M \backslash \gamma$. Recall that the singular values $s_{j}(y), j=$ $1,2,3$ of $D F(y)$ are the square roots of the eigenvalues of $(D F(y))^{t} D F(y)$, where $(D F)^{t}$ is the transpose of $D F$. By (17), the singular values $s_{j}=s_{j}(y)$, $j=1,2,3$, of $D F(y)$, numbered in increasing order, satisfy

$$
\begin{aligned}
& c_{1} \leq s_{1}(y) \leq c_{2}, \\
& c_{1} \leq s_{2}(y) \leq c_{2}, \\
& \frac{c_{1}}{\tau(y)} \leq s_{3}(y) \leq \frac{c_{2}}{\tau(y)},
\end{aligned}
$$

where $c_{1}, c_{2}>0$.

The determinant of the matrix $D F(y)$ can be computed in terms of its singular values by $\operatorname{det}(D F)=s_{1} s_{2} s_{3}$. Later, we need the norm of the matrix 
$\operatorname{det}(D F(y))^{-1} D F(y)$. It satisfies by formula (8)

$$
\left\|\operatorname{det}(D F(y))^{-1} D F(y)\right\|=\left\|\left(\prod_{k=1}^{3} s_{k}^{-1}\right) \operatorname{diag}\left(s_{1}, s_{2}, s_{3}\right)\right\|=\max _{1 \leq j \leq 3} \prod_{k \neq j} s_{k}^{-1} \leq c_{1}^{-2} .
$$

\subsection{Maxwell's equations on the wormhole with SH coat- ing}

Let $d V_{0}(x)$ denote the Euclidian volume element on $\bar{N} \subset \mathbb{R}^{3}$. Recall that $N \subset \mathbb{R}^{3}$ is open set with boundary $\partial N=\Sigma$. Let $d V_{g}$ be the Riemannian volume on $(M, g)$. We consider below the map $F: M \backslash \gamma \rightarrow N$ as a coordinate deformation. The map $F$ induces for any differential form $\widetilde{E}$ on $N$ a form $E=F^{*} \widetilde{E}$ in $M \backslash \gamma$ called the pull back of $\widetilde{E}$ in $F$, see [2].

Next, we consider Maxwell equations with degenerate material parameters $\widetilde{\varepsilon}$ and $\widetilde{\mu}$ on $N$ with SH boundary conditions on $\Sigma$. On $M$ and $N$ we define the permittivity and permeability by setting

$$
\begin{aligned}
& \varepsilon^{j k}=\mu^{j k}=\operatorname{det}(g)^{1 / 2} g^{j k}, \quad \text { on } M, \\
& \widetilde{\varepsilon}^{j k}=\widetilde{\mu}^{j k}=\operatorname{det}(\widetilde{g})^{1 / 2} \widetilde{g}^{j k}, \quad \text { on } N \text {. }
\end{aligned}
$$

Here, and below, the matrix $\left[g_{j k}(x)\right]$ is the representation of the metric $g$ in local coordinates, $\left[g^{j k}(x)\right]$ is the inverse of the matrix $\left[g_{j k}(x)\right]$, and $\operatorname{det}(g)$ is the determinant of $\left[g_{j k}(x)\right]$. We note that the metric $\widetilde{g}$ is degenerate near $\Sigma$, and thus $\widetilde{\varepsilon}$ and $\widetilde{\mu}$, represented as matrices in the Euclidian coordinates, have elements that tend to infinity at $\Sigma$, that is, the matrices $\widetilde{\varepsilon}$ and $\widetilde{\mu}$ have a singularity near $\Sigma$.

Remark 1. Modifying the above construction by replacing $M_{2}$ with $M_{2}=$ $\mathbb{S}_{\text {flat }}^{2} \times\left[l_{1}, l_{2}\right]$ for appropriate $l_{1}, l_{2} \in \mathbb{R}$ and choosing $F_{1}$ in an appropriate way, we can use local coordinates $(\widetilde{\theta}, \widetilde{t})$ on $\Sigma$ such that the Euclidian distance along $\Sigma$ of points $\left(\widetilde{\theta}, \widetilde{t}_{1}\right)$ and $\left(\widetilde{\theta}, \widetilde{t}_{2}\right)$ is proportional to $\left|\widetilde{t}_{1}-\widetilde{t}_{2}\right|$, and the metric $\widetilde{g}$ in the Euclidian boundary normal coordinates $(\widetilde{\theta}, \widetilde{t}, \widetilde{\tau})$ associated to $\partial K$ has the form

$$
d s^{2}=4 d \widetilde{\tau}^{2}+d \widetilde{t}^{2}+4 \widetilde{\tau}^{2} d \widetilde{\theta}^{2}, \quad 0<\widetilde{\tau}<\frac{1}{4} .
$$

The metric corresponding to the metamaterials used in the physical experiment in [20] has the same form in Euclidian boundary normal coordinates 
associated to an infinitely long cylinder $B_{2}(0,1) \times \mathbb{R}$. Thus it seems likely that metamaterials similar to those used in the experimental verification of cloaking could be used to create physical wormhole devices working at microwave frequencies.

\subsection{Finite energy solutions of Maxwell's equations and the equivalence theorem}

In the following, we consider 1-forms $\widetilde{E}=\sum_{j} \widetilde{E}_{j} d \widetilde{x}^{j}$ and $\widetilde{H}=\sum_{j} \widetilde{H}_{j} d \widetilde{x}^{j}$ in the Euclidian coordinates $\left(\widetilde{x}^{1}, \widetilde{x}^{2}, \widetilde{x}^{3}\right)$ of $N \subset \mathbb{R}^{3}$. In the sequel, we use Einstein's summation convention and omit the sum signs. We use the Euclidian coordinates as we want to consider $N$ with the differential structure inherited from the Euclidian space. We say that $\widetilde{E}_{j}$ and $\widetilde{H}_{j}$ are the (Euclidian) coefficients of the forms $\widetilde{E}$ and $\widetilde{H}$, correspondingly. We say that these coefficients are in $L_{l o c}^{p}\left(\bar{N}, d V_{0}\right), 1 \leq p<\infty$, if

$$
\int_{W}\left|E_{j}(x)\right|^{p} d V_{0}(x)<\infty, \quad \text { for all bounded measurable sets } W \subset \bar{N} .
$$

Definition 4.1 We say that the 1-forms $\widetilde{E}$ and $\widetilde{H}$ are finite energy solutions of Maxwell's equations in $N$ with the soft-and-hard (SH) boundary conditions on $\Sigma$ and the frequency $k \neq 0$,

$$
\begin{aligned}
& \nabla \times \widetilde{E}=i k \widetilde{\mu}(x) \widetilde{H}, \quad \nabla \times \widetilde{H}=-i k \widetilde{\varepsilon}(x) \widetilde{E}+\widetilde{J} \quad \text { on } N, \\
& \left.\widetilde{\eta} \cdot \widetilde{E}\right|_{\Sigma}=0,\left.\quad \widetilde{\eta} \cdot \widetilde{H}\right|_{\Sigma}=0,
\end{aligned}
$$

if 1-forms $\widetilde{E}$ and $\widetilde{H}$ and 2-forms $\widetilde{D}=\widetilde{\varepsilon} \widetilde{E}$ and $\widetilde{B}=\widetilde{\mu} \widetilde{H}$ in $N$ have coefficients in $L_{\mathrm{loc}}^{1}\left(\bar{N}, d V_{0}\right)$ and satisfy

$$
\begin{aligned}
\|\widetilde{E}\|_{L^{2}\left(W,|\widetilde{g}|^{1 / 2} d V_{0}\right)}^{2} & =\int_{W} \widetilde{\varepsilon}^{j k} \widetilde{E}_{j} \overline{\widetilde{E}_{k}} d V_{0}(x)<\infty, \\
\|\widetilde{H}\|_{\left.L^{2}\left(W,|\widetilde{g}|^{1 / 2} d V_{0}\right)\right)}^{2} & =\int_{W} \widetilde{\mu}^{j k} \widetilde{H}_{j} \overline{\widetilde{H}_{k}} d V_{0}(x)<\infty
\end{aligned}
$$

for all bounded measurable sets $W \subset N$, and finally,

$$
\begin{aligned}
& \int_{N}((\nabla \times \widetilde{h}) \cdot \widetilde{E}-i k \widetilde{h} \cdot \widetilde{\mu}(x) \widetilde{H}) d V_{0}(x)=0, \\
& \int_{N}((\nabla \times \widetilde{e}) \cdot \widetilde{H}+\widetilde{e} \cdot(i k \widetilde{\varepsilon}(x) \widetilde{E}-\widetilde{J})) d V_{0}(x)=0,
\end{aligned}
$$


for all 1-forms $\widetilde{e}$ and $\widetilde{h}$ with coefficients in $C_{0}^{\infty}(\bar{N})$ that satisfy

$$
\left.\widetilde{\eta} \cdot \widetilde{e}\right|_{\Sigma}=0,\left.\quad \widetilde{\eta} \cdot \widetilde{h}\right|_{\Sigma}=0,
$$

where $\widetilde{\eta}=\partial_{\theta}$ is the angular vector field that is tangential to $\Sigma$.

Below, we use for 1-forms $E=E_{j} d x^{j}$ and $H=H_{j} d x^{j}$, given in local coordinates $\left(x^{1}, x^{2}, x^{3}\right)$ on $M$, the notations

$$
\nabla \times E=d H, \quad \nabla \cdot(\varepsilon E)=d * E, \quad \nabla \cdot(\mu H)=d * H,
$$

where $d$ is the exterior derivative and $*$ is the Hodge operator on $(M, g)$, cf. formula (10).

We have the following "equivalent behavior of electromagnetic fields on $N$ and $M$ " result, analogous to the results of [3] for cloaking.

Theorem 4.2 Let $E$ and $H$ be 1-forms on $M \backslash \gamma$ and $\widetilde{E}$ and $\widetilde{H}$ be 1-forms with coefficients in $L_{\mathrm{loc}}^{1}\left(\bar{N}, d V_{0}\right)$ such that $E=F^{*} \widetilde{E}, H=F^{*} \widetilde{H}$. Let $\widetilde{J}$ and $J=F^{*} \widetilde{J}$ be 2-forms with smooth coefficients in $N$ and $M \backslash \gamma$ that are supported away from $\Sigma$ and $\gamma$.

Then the following are equivalent:

1. On $N$, the 1-forms $\widetilde{E}$ and $\widetilde{H}$ satisfy Maxwell's equations with SH boundary conditions in the sense of Definition 4.1 .

2. On $M$, the forms $E$ and $H$ can be extended on $M$ so that they are classical solutions $E$ and $H$ of Maxwell's equations,

$$
\begin{aligned}
& \nabla \times E=i k \mu H, \quad \text { in } M, \\
& \nabla \times H=-i k \varepsilon E+J, \quad \text { in } M .
\end{aligned}
$$

Proof. Assume first that $E$ and $H$ satisfy Maxwell's equations on $M$ with source $J$ supported away from $\gamma$. Then $E$ and $H$ are $C^{\infty}$ smooth near $\gamma$.

Using $F^{-1}: N \rightarrow M \backslash \gamma$ we define the 1-forms $\widetilde{E}, \widetilde{H}$ and 2-form $\widetilde{J}$ on $N$ by $\widetilde{E}=\left(F^{-1}\right)^{*} E, \widetilde{H}=\left(F^{-1}\right)^{*} H$, and $\widetilde{J}=\left(F^{-1}\right)^{*} J$. These fields satisfy Maxwell's equations in $N$,

$$
\nabla \times \widetilde{E}=i k \widetilde{\mu}(x) \widetilde{H}, \quad \nabla \times \widetilde{H}=-i k \widetilde{\varepsilon}(x) \widetilde{E}+\widetilde{J} \quad \text { in } N .
$$


Now, writing $E=E_{j}(x) d x^{j}$ on $M$ near $\gamma$, we see using the transformation rule for differential 1-forms that the form $\widetilde{E}=\left(F^{-1}\right)^{*} E$ is in local coordinates

$$
\widetilde{E}=\widetilde{E}_{j}(\widetilde{x}) d \widetilde{x}^{j}, \quad \widetilde{E}_{j}(\widetilde{x})=\left(D F^{-1}\right)_{j}^{k}(\widetilde{x}) E_{k}\left(F^{-1}(\widetilde{x})\right), \quad \widetilde{x} \in N
$$

Using the smoothness of $E$ and $H$ near $\gamma$ on $M$ and formulae (7), we see that $\widetilde{E}, \widetilde{H}$ are forms on $N$ with $L_{\text {loc }}^{1}\left(\bar{N}, d V_{0}\right)$ coefficients. Moreover,

$$
\begin{aligned}
\widetilde{\varepsilon}(x) \widetilde{E}(x) & =\operatorname{det}(D F(y))^{-1} D F(y) \varepsilon(y) D F(y)^{t}\left(D F(y)^{t}\right)^{-1} E(y) \\
& =\operatorname{det}(D F(y))^{-1} D F(y) \varepsilon(y) E(y)
\end{aligned}
$$

where $x \in N, y=F^{-1}(x) \in M \backslash \gamma$. Formula (9) shows that $\widetilde{D}=\widetilde{\varepsilon} \widetilde{E}$, and $\widetilde{B}=\widetilde{\mu} \widetilde{H}$ are 2 -forms on $N$ with $L_{\text {loc }}^{1}\left(\bar{N}, d V_{0}\right)$ coefficients.

Let $\Sigma(t) \subset \bar{N}$ be the $t$-neighbourhood of $\Sigma$ in the $\widetilde{g}$-metric. Note that for small $t>0$ the set $\Sigma(t)$ is the Euclidian $(t / 2)$-neighborhood of $\partial K$. Denote by $\nu$ be the unit exterior Euclidian normal vector of $\partial \Sigma(t)$ and the Euclidian inner product by $(\widetilde{\eta}, \widetilde{E})_{g^{e}}=\widetilde{\eta} \cdot \widetilde{E}$.

Formulas (17) and (13) imply that the angular components satisfy

$$
|\widetilde{\eta} \cdot \widetilde{E}| \leq C t, \quad x \in \partial \Sigma(t)
$$

and

$$
|\widetilde{\zeta} \cdot \widetilde{E}| \leq C, \quad x \in \partial \Sigma(t)
$$

with some $C>0$. Thus denoting by $d S$ the Euclidian surface area on $\partial \Sigma(t)$, Stokes' formula, formula (12), and the identity $\nu \times \widetilde{\xi}= \pm \widetilde{\eta}$ yield

$$
\begin{aligned}
& \int_{N}((\nabla \times \widetilde{h}) \cdot \widetilde{E}-i k \tilde{h} \cdot \widetilde{\mu} \widetilde{H}) d V_{0}(x) \\
& =\lim _{t \rightarrow 0} \int_{N \backslash \Sigma(t)}((\nabla \times \widetilde{h}) \cdot \widetilde{E}-i k \widetilde{h} \cdot \widetilde{\mu} \widetilde{H}) d V_{0}(x) \\
& =-\lim _{t \rightarrow 0} \int_{\partial \Sigma(t)}(\nu \times \widetilde{E}) \cdot \widetilde{h} d S(x) \\
& =-\lim _{t \rightarrow 0} \int_{\partial \Sigma(t)} \nu \times((\widetilde{\eta} \cdot \widetilde{E}) \widetilde{\eta}+(\widetilde{\zeta} \cdot \widetilde{E}) \widetilde{\zeta}) \cdot \widetilde{h} d S(x) \\
& =0
\end{aligned}
$$


for a test function $\widetilde{h}$ satisfying formula (11).

Similar analysis for $\widetilde{H}$ shows that 1-forms $\widetilde{E}$ and $\widetilde{H}$ satisfy Maxwell's equations with $\mathrm{SH}$ boundary conditions in the sense of Definition 4.1.

Next, assume that $\widetilde{E}$ and $\widetilde{H}$ form a finite energy solution of Maxwell's equations on $(N, g)$ with a source $\widetilde{J}$ supported away from $\Sigma$, implying in particular that

$$
\widetilde{\varepsilon}^{j k} \widetilde{E}_{j}{\widetilde{E_{k}}} \in L^{1}\left(W, d V_{0}\right), \quad \widetilde{\mu}^{j k} \widetilde{H}_{j}{\widetilde{\tilde{H}_{k}}}_{\in} \in L^{1}\left(W, d V_{0}\right)
$$

where $W=F(U \backslash \gamma) \subset N$ and $U \subset M$ is a relatively compact open neighbourhood of $\gamma, \operatorname{supp}(\widetilde{J}) \cap W=\emptyset$. Define $E=F^{*} \widetilde{E}, H=F^{*} \widetilde{H}$, and $J=F^{*} \widetilde{J}$ on $M \backslash \gamma$. Therefore we conclude that

$$
\nabla \times E=i k \mu(x) H, \quad \nabla \times H=-i k \varepsilon(x) E+J, \quad \text { in } M \backslash \gamma
$$

and

$$
\varepsilon^{j k} E_{j} \overline{E_{k}} \in L^{1}\left(U \backslash \gamma, d V_{g}\right), \quad \mu^{j k} H_{j} \overline{H_{k}} \in L^{1}\left(U \backslash \gamma, d V_{g}\right)
$$

As representations of $\varepsilon$ and $\mu$, in local coordinates of $M$, are matrices that are bounded from above and below, these imply that

$$
\begin{aligned}
& \nabla \times E \in L^{2}\left(U \backslash \gamma, d V_{g}\right), \quad \nabla \times H \in L^{2}\left(U \backslash \gamma, d V_{g}\right), \\
& \nabla \cdot(\varepsilon E)=0, \quad \nabla \cdot(\mu H)=0, \quad \text { in } U \backslash \gamma .
\end{aligned}
$$

Let $E^{e}, H^{e} \in L^{2}\left(U, d V_{g}\right)$ be measurable extensions of $E$ and $H$ to $\gamma$. Then

$$
\begin{aligned}
& \nabla \times E^{e}-i k \mu(x) H^{e}=0, \quad \text { in } U \backslash \gamma, \\
& \nabla \times E^{e}-i k \mu(x) H^{e} \in H^{-1}\left(U, d V_{g}\right), \\
& \nabla \times H^{e}+i k \varepsilon(x) E^{e}=0, \quad \text { in } U \backslash \gamma, \\
& \nabla \times H^{e}+i k \varepsilon(x) E^{e} \in H^{-1}\left(U, d V_{g}\right),
\end{aligned}
$$

where $H^{-1}\left(U, d V_{g}\right)$ is the Sobolev space with smoothness $(-1)$ on $(U, g)$. Since $\gamma$ is a subset with (Hausdorff) dimension 1 of the 3-dimensional domain $U$, it has zero capacitance. Thus, the Lipschitz functions on $U$ that vanish on $\gamma$ are dense in $H^{1}(U)$, see [12]. Therefore, there are no non-zero distributions in $H^{-1}(U)$ supported on $\gamma$. Hence we see that

$$
\nabla \times E^{e}-i k \mu(x) H^{e}=0, \quad \nabla \times H^{e}+i k \varepsilon(x) E^{e}=0 \quad \text { in } U .
$$


This also implies that

$$
\nabla \cdot\left(\varepsilon E^{e}\right)=0, \quad \nabla \cdot\left(\mu H^{e}\right)=0 \quad \text { in } U,
$$

which, by elliptic regularity, imply that $E^{e}$ and $H^{e}$ are $C^{\infty}$ smooth in $U$.

In summary, $E$ and $H$ have unique continuous extensions to $\gamma$, and the extensions are classical solutions to Maxwell's equations.

\section{References}

[1] G. Eleftheriades and K. Balmain, Negative-Refraction Metamaterials, IEEE Press (Wiley-Interscience), 2005.

[2] T. Frankel, The geometry of physics, Cambridge University Press, Cambridge, 1997.

[3] A. Greenleaf, Y. Kurylev, M. Lassas and G. Uhlmann, Full-wave invisibility of active devices at all frequencies, ArXiv.org:math.AP/0611185), 2006; Comm. Math. Phys., to appear.

[4] A. Greenleaf, Y. Kurylev, M. Lassas and G. Uhlmann, Electromagnetic wormholes and virtual magnetic monopoles, ArXiv.org:math-ph/0703059, submitted, 2007.

[5] A. Greenleaf, M. Lassas, and G. Uhlmann, The Calderón problem for conormal potentials, I: Global uniqueness and reconstruction, Comm. Pure Appl. Math 56 (2003), no. 3, 328-352

[6] A. Greenleaf, M. Lassas, and G. Uhlmann, Anisotropic conductivities that cannot detected in EIT, Physiological Measurement (special issue on Impedance Tomography), 24 (2003), pp. 413-420.

[7] A. Greenleaf, M. Lassas, and G. Uhlmann, On nonuniqueness for Calderón's inverse problem, Math. Res. Let. 10 (2003), no. 5-6, 685-693.

[8] I. Hänninen, I. Lindell, and A. Sihvola, Realization of generalized Softand-Hard Boundary, Progr. In Electromag. Res., PIER 64, 317-333, 2006.

[9] S. Hawking and G. Ellis, The Large Scale Structure of Space-Time, Cambridge Univ. Press, 1973. 
[10] P.-S. Kildal, Definition of artificially soft and hard surfaces for electromagnetic waves, Electron. Lett. 24 (1988), 168-170.

[11] P.-S. Kildal, Artificially soft-and-hard surfaces in electromagnetics, IEEE Trans. Ant. and Propag., 10 (1990), 1537-1544.

[12] T. Kilpeläinen, J. Kinnunen, and O. Martio, Sobolev spaces with zero boundary values on metric spaces. Potential Anal. 12 (2000), no. 3, 233247.

[13] R. Kohn, H. Shen, M. Vogelius, and M. Weinstein, in preparation.

[14] Y. Kurylev, M. Lassas, and E. Somersalo, Maxwell's equations with a polarization independent wave velocity: Direct and inverse problems, $J$. Math. Pures Appl., 86 (2006), 237-270.

[15] M. Lassas, M. Taylor, G. Uhlmann, On determining a non-compact Riemannian manifold from the boundary values of harmonic functions, Comm. Geom. Anal. 11 (2003), 207-222.

[16] U. Leonhardt, Optical Conformal Mapping, Science 312 (23 June, 2006), $1777-1780$

[17] G. Milton, M. Briane, J. Willis, On cloaking for elasticity and physical equations with a transformation invariant form, New J. Phys. 8 (2006), 248.

[18] J.B. Pendry, D. Schurig, D.R. Smith, Controlling Electromagnetic Fields, Science 312 (23 June, 2006), 1780-1782.

[19] J.B. Pendry, D. Schurig, D.R. Smith, Optics Express 14, 9794 (2006).

[20] D. Schurig, J. Mock, B. Justice, S. Cummer, J. Pendry, A. Starr, and D. Smith, Metamaterial electromagnetic cloak at microwave frequencies, Science 314 (10 Nov. 2006), 977-980.

[21] R. Weder, A rigorous time-domain analysis of full-wave electromagnetic cloaking (Invisibility), preprint, ArXiv.org:07040248v1 (2007).

[22] M. Visser, Lorentzian Wormholes, AIP Press, 1997. 
Department of Mathematics

UNIVERSITY OF ROCHESTER

Rochester, NY 14627, USA, allan@math.rochester.edu

Department of Mathematical Sciences

UNIVERSITY OF LOUGHBOROUGH

LoughBorough, LE11 3TU, UK, Y.V.Kurylev@lboro.ac.uk

Institute of MATHEMATICS

Helsinki University of Technology

EsPoo, FIN-02015, Finland, Matti.Lassas@tkk.fi

Department of Mathematics

UNIVERSITY OF WASHINGTON

SEATtLe, WA 98195, USA, gunther@math.washington.edu 